\begin{tabular}{|c|l|}
\hline Title & Mass transfer caused by gravitational instability at reactive solid-liquid interfaces \\
\hline Author(s) & Otomo, Ryoko; Ishii, Nobuhiko; Takahashi, Keita; Harada, Shusaku \\
\hline Citation & $\begin{array}{l}\text { Journal of Visualization, 17(1), 49-57 } \\
\text { https://doi.org/40.1007/S12650-013-0183-0 }\end{array}$ \\
\hline Issue Date & 201402 \\
\hline Doc URL & http://hdl.handle.net/2115/57887 \\
\hline Rights & The final publication is available at Springer via http://dx.doi.org/10.1007/s12650-013-0183-0 \\
\hline Type & article (author version) \\
\hline File Information & JVis.17(1).49-57.pdf \\
\hline
\end{tabular}

Instructions for use 


\title{
Mass Transfer caused by Gravitational Instability at Reactive Solid-liquid Interfaces
}

\author{
Ryoko Otomo \\ Faculty of Engineering, Hokkaido University, N13-W8, Sapporo, 0608628, Japan \\ Research Fellow of the Japan Society for the Promotion of Science \\ Email: ootomo@trans-er.eng.hokudai.ac.jp \\ Phone: +81117066311 \\ Fax: +81117066311
}

Nobuhiko Ishii

Faculty of Engineering, Hokkaido University, N13-W8, Sapporo, 0608628, Japan

Keita Takahashi

Faculty of Engineering, Hokkaido University, N13-W8, Sapporo, 0608628, Japan

\author{
Shusaku Harada \\ Faculty of Engineering, Hokkaido University, N13-W8, Sapporo, 0608628, Japan
}

\begin{abstract}
Mass transfer in porous media has been investigated experimentally. In this paper, we present a visualization technique and discuss the behavior of a substance which transfers under the influence of gravity and reacts with the surface of porous media. Mass transfer by the reaction with porous media was demonstrated by means of electrochemical deposition experiment on particulate beds with complex structures. A copper plate (anode) and a stainless steel particulate bed (cathode) were respectively placed at the upper and bottom side of a thin vertical cell which was filled with copper sulfate solution. After the application of electricity, cupric ion which is provided from the copper plate to the solution transfers under the influence of gravity and it is consumed by deposition at the particulate bed. The behavior of ions between the electrodes was visualized by utilizing the infrared absorption characteristics of cupric ion. We observed gravitational instability and convection flow due to concentration gradient of ions in opposite direction to that of gravity, which is formed by reaction at solid-liquid interfaces. While downward flow caused by RayleighTaylor instability was observed in the case of flat interfaces, upward flow generated from complex-shaped interfaces was greatly dependent on their geometry. The interaction of these flows resulted in the convection throughout the cell. Consequently, it is found from the results that the gravitational instability significantly varies the transport characteristics and that the reactive interface geometry greatly affects the overall mass transfer.
\end{abstract}


Keywords: Mass transfer, Reactive solid-liquid interface, Porous media, Gravitational instability, Convection flow

\section{Introduction}

Mass transfer in porous media is of great interest in various engineering processes such as filtration, adsorption, and contamination control in the media. These processes require the mass transfer characteristics for promotion of the efficiency and optimization of the system. However, the transfer of substances in porous media is difficult to understand because the transport is in many cases affected by the fluid convection that is sensitive to ambient conditions such as complicated porous geometry or effects of the gravity.

One of the most influential factors on the convective flow is reaction. Particularly, the reaction of substances with the porous media surface varies the shape of the porous surface, namely the geometry of flow channel, and consequently it affects the transport characteristics in the media with time. A number of researchers have studied the transport properties in porous media associated with change in the porous geometry by the reaction (Békri et al. 1995; Borisova and Adler 2005; Colón et al. 2004; Sahimi et al. 1990; Sallès et al. 1993; Schechter and Gidley 1969). For example, Colón et al. (2004) experimentally investigated subsequent changes of fluid permeability and reactive surface area in rocks where the pore structure varies due to the dissolution by acidic fluid. On the other hand, Borisova and Adler (2005) examined numerically the transport properties during deposition and clogging in porous media, and found their dependence on initial porosity and structure of the media.

The importance of the gravity to mass transfer in porous media has been also recognized (Ennis-King and Paterson 2007; Hinch and Bhatt 1990; Riaz et al. 2006). Ennis-King and Paterson (2007) studied the transfer of dissolved carbon dioxide in geological storage. They numerically investigated the density instability and convective behavior of $\mathrm{CO}_{2}$-rich (denser) brine which is formed on pure (lighter) brine. From these studies, it is noted that the density instability affects the convective flow and consequently it varies the transport characteristics in the overall system. 
Previous studies on mass transfer in porous media have been interested in the evaluation of the macroscopic transport properties inside the media. However, the mass transfer inside the media would be affected by that in the entrance part. For instance, the clogging of the fluid channel at the end of the media prevents the overall transport inside them. Therefore, it would be more important to understand the transfer at the end of the media in order to evaluate accurate transport characteristics. Li et al. (2008) investigated the structural evolution in the vicinity of the end of porous media during mineral precipitation. Nevertheless, to our knowledge, there has been no research which focuses on effects of initial geometry at the end of porous media on overall mass transfer.

The purpose of this study is to investigate mass transfer in the vicinity of the reactive solid-liquid interfaces having complicated geometry as shown in Fig. 1 and effects of the initial geometry of the interfaces on overall mass transfer. In this paper, we present a visualization technique of ion behavior by means of light absorption characteristics and discuss from observation results the mass transfer in the presence of convection by the gravitational instability. The electrochemical deposition experiment is employed as one kind of mass transfer system with the change in pore geometry. It allows us to observe the change of geometry at the end of porous media whereas the actual mineral precipitation requires enormous amount of years to evolve. Although our mass transfer system is based on electrochemical ones, it can be apparently interpreted as one case of advectiondiffusion system without the electricity (Newman and Thomas-Alyea 2004; Nicoli et al. 2008). In the electroneutral region which occupies most of the system, ion transfer is described by advection-diffusion equation with the apparent diffusion coefficient which represents that the effect of electric potential apparently disappears as a result of the interaction between migration and diffusion. The boundary flux at the solid-liquid interface is expressed in the similar form to nonelectrolytic system, i.e., the multiplication of the concentration and reaction rate. Only the reaction rate depends on the electric potential, which would not affect the convective flow caused by the gravitational instability. 


\section{Experimental Methodology}

\section{1 Electrochemical deposition of copper on particulate bed}

Mass transfer in porous media is demonstrated by electrochemical deposition experiments of copper ion to particulate beds. Figure 2 shows our experimental setup. A vertical quasi-two-dimensional deposition cell is prepared as shown in Fig. 2(a). The cell has a length $l=55 \mathrm{~mm}$, a height $h=30 \mathrm{~mm}$ and a width $w=3 \mathrm{~mm}$ and it is filled with $1.0 \mathrm{~mol} / \mathrm{L}$ copper sulphate solution and $0.5 \mathrm{~mol} / \mathrm{L}$ sulphuric acid. A flat copper plate is placed at the top side of the cell, while stainless steel particles (diameter $d_{p}=2 \mathrm{~mm}$ ) are placed at the bottom side. When the electricity is applied, copper plate acts as anode and it dissolve cupric ion into the solution while cupric ion in the solution deposits on the surface of the stainless particulate bed which works as cathode. The current $I$ is maintained to be constant (galvanostatic conditions) and $I=36 \mathrm{~mA}$. During the application of the electricity for 60 hours, we take images of deposition behavior on the surface of the particulate bed every 12 hours. From these images, we infer the mass transfer of cupric ion in the overall cell with reaction at the surface of the particulate bed.

\subsection{Visualization of cupric ion behavior by absorption technique}

The ion behavior in liquid was visualized by utilizing the infrared (IR) absorption characteristics of cupric ion. This method was originally proposed by Rosso et al. (1994). Although this visualization technique in principle enables us to obtain the quantitative profile of ionic concentration, the qualitative behavior of the cupric ion is shown and discussed in the present study.

Figure 2(b) shows the apparatus of the visualization experiments. We employed the deposition cell with a length $l=20 \mathrm{~mm}$, a height $h=10 \mathrm{~mm}$ and a width $w=1 \mathrm{~mm}$, which is filled with $1.0 \mathrm{~mol} / \mathrm{L}$ copper sulphate solution. A copper plate and stainless steel particles (diameter $d_{p}=1 \mathrm{~mm}$ ) are placed in the same way as presented in Fig. 2(a). 5mA current is applied for 24 hours. During the depositions, the IR light (wavelength more than $800 \mathrm{~nm}$ ) is irradiated to the cell from backward and the images of the inside of the cell are taken from the front side. Since the peak of the absorption wavelength for the cupric ion exists around $810 \mathrm{~nm}$ as shown in Fig. 3, the solution in the cell exhibits the non-uniform 
brightness depending on the local ionic concentration. In other words, the part of high concentration appears to be dark in the image since the ion absorbs the IR light much more, while the part of low concentration becomes bright because there are only a little of ion which absorbs the light. We take images of twodimensional brightness variances and observe the convective behavior of cupric ion at the initial and steady stage in the experiment. The images are modified in two steps. Firstly, we subtract a reference image which is taken prior to the experiment in order to calibrate the IR light intensity. Then, the contrast of the brightness in the images is enhanced so that we could see the ion behavior more clearly.

\section{Results and discussion}

\subsection{Transport of cupric ion to particulate bed during deposition}

The effect of initial geometry of the end of porous media on mass transfer has been discussed from the results of the deposition experiments of cupric ion on different particulate beds. Figure 4 shows the photographs of three kinds of particulate bed as examples of complicate-shaped interface. The particulate bed in Fig. 4(a) represents a simple and gentle concavo-convex structure and it is called "standard case" in this paper. The other two structures in Fig. 4(b) and Fig. 4(c) represent "comb-shaped" and "cove-shaped", respectively. We observe shape evolution of the particulate bed in the deposition process and discuss the effect of the interface geometry on the ion transfer, namely the mass transfer.

After the experiments for 60 hours, the copper deposits to each surface as shown in the second row in Fig. 4. For the standard case (Fig. 4a), it is found that the copper locally deposits to particles with almost keeping their spherical shape. However, the overall deposition is not uniform and it is likely to occur preferentially at the point where the particles are less surrounded by others. Such selective growth of the deposits is also shown for the other geometries. For the comb-shaped (Fig. 4b) and cove-shaped (Fig. 4c) structures, the convex particles have more deposits and the surface in the back of the cove less grows up.

In order to investigate how the ion transfer is related to this selective growth, we illustrate the contour of deposits every 12 hours as shown in the bottom row in 
Fig. 4. For the comb-shaped and cove-shaped cases, the deposition in the back of the cove appears to decrease as the surface in the inlet of the cove grows. These results indicate that the surface growth in the inlet of the cove affected the ion transfer and the electrochemical state, and consequently the deposition in the back of the cove is suppressed. Therefore, it would be inferred that the surface geometry at the end of the porous media affects inward mass transfer.

Figure 5 exhibits the ratio of the liquid part to the solid part for various heights of the cell. The ratio was obtained from the length of horizontal lines drawn at each height in photographs of the particulate bed for every 12 hours. For standard structure shown in Fig. 5(a), the liquid part at each height decreases as time passes due to the growth of solid surface by deposition. On the other hand, S-curve is observed for comb-shaped and cove-shaped structures because the cove has clogged in the process of the deposition. These results indicate that initial geometry of particulate bed would affect the ion transfer and consequently the selective growth of the interfaces. As a result, it is inferred that the mass transfer in the vicinity of reactive solid-liquid interfaces is related to their initial geometry.

\subsection{Visualization of convective behavior of cupric ion}

\subsubsection{Gravitational instability}

Prior to the discussion of the results, we refer to Rayleigh-Taylor instability in this section. When the denser fluid exists on the dilute one, their interface becomes instable due to the gravity and consequently the convective flow arises. The gravitational instability had been investigated at the interface of both miscible and immiscible fluids (Fernandez et al. 2001). The lengthscale and the growth rate of the instability can be calculated by linear stability analysis. In quasi-twodimensional system, the interface geometry is described by the superposition of waves having various wavelengths as follows.

$$
z=z_{0} \exp (n t) \exp (i k x) \zeta(y)
$$

where $z$ is the perturbation of the interface, $z_{0}$ is a constant, $k$ is the wave number, $n$ is the growth rate, and $\zeta$ is the Poiseuille factor which expresses the interface in

$y$ (quasi-two-dimensional) direction. As the instable progresses, only the wave having a certain length becomes larger in amplitude and the other waves decay 
gradually. This length is called dominant wavelength and obtained from theoretical calculation. For immiscible fluids with no interfacial tension (or miscible fluids with no diffusion), quasi-two-dimensional Stokes equation with perturbation terms results in the following dispersion relation (Harada et al. 2012; Völtz 2003).

$$
\begin{gathered}
-\left[\frac{g k}{n^{2}}\left(\alpha_{1}-\alpha_{2}\right)+\beta_{1}+\beta_{2}\right]\left[\beta_{2} q_{1}+\beta_{1} q_{2}-k\left(\beta_{1}+\beta_{2}\right)\right]-4 k \beta_{1} \beta_{2} \\
+\frac{4 k^{2}}{n}\left(\alpha_{1} \overline{v_{1}}-\alpha_{2} \bar{v}_{2}\right)\left[\beta_{2} q_{1}-\beta_{1} q_{2}+k\left(\beta_{1}-\beta_{2}\right)\right] \\
+\frac{4 k^{3}}{n^{2}}\left(\alpha_{1} \bar{v}_{1}-\alpha_{2} \bar{v}_{2}\right)^{2}\left(q_{1}-k\right)\left(q_{2}-k\right)=0
\end{gathered}
$$

where $\bar{\rho}_{1}, \overline{v_{1}}$ is the density and kinetic viscosity of the lower (dilute) fluid, $\bar{\rho}_{2}$, $\overline{v_{2}}$ is those of the upper (denser) fluid respectively, the other parameters are defined as $\alpha_{1}=\bar{\rho}_{1} /\left(\overline{\rho_{1}}+\bar{\rho}_{2}\right), \quad \alpha_{2}=\bar{\rho}_{2} /\left(\overline{\rho_{1}}+\bar{\rho}_{2}\right), \quad \beta_{1}=\alpha_{1}\left(1+\overline{\nu_{1}} \eta / n\right)$, $\beta_{2}=\alpha_{2}\left(1+\overline{v_{2}} \eta / n\right) \quad, \quad q_{1}=\sqrt{n / \overline{\nu_{1}}+k^{2}+\eta} \quad, \quad q_{2}=\sqrt{n / \overline{v_{2}}+k^{2}+\eta} \quad$ and $\eta=-\partial^{2} \zeta / \partial y^{2}=12 / D^{2} . D$ is the depth in $y$ direction. Our experimental system includes the gravitational instability at the interface of two miscible fluids, namely higher and lower concentration copper sulphate solution. If the diffusion can be neglected, we can obtain from Eq. (2) the wavelength against the growth rate in our experimental cell as shown in Fig. 6. The dominant wave number is $k_{\max }=$ $3.709 \mathrm{~mm}^{-1}$ and consequently dominant wavelength is $\lambda_{\max }=2 \pi / k_{\max }=1.694 \mathrm{~mm}$.

\subsubsection{Visualization of ion behavior caused by gravitational instability at reactive solid-liquid interfaces}

We have performed the visualization by utilizing the absorption characteristics and observed the ion behavior in the process of the electrochemical deposition experiments. It is considered in the experiments that the ion is transported to the particulate bed by convective motion of the ambient fluid which results from the gravitational instability. In our deposition experiments, cupric ion is provided from the top (copper plate) into the solution and it is consumed by deposition at the bottom (particulate bed) when the reaction occurs at the surface of the copper plate and the particulate bed. Therefore, the high-concentration and low- 
concentration regions are generated at the top and the bottom of the cell, respectively. Consequently, the concentration gradient in the opposite direction to the gravity brings about the gravitational instability as described in the previous section.

To observe these ion behavior caused by gravitational instability at reactive solid-liquid interfaces, we have started with the experiments in which both of reactive surfaces consist of copper plates, i.e., the solid-liquid interfaces have not complicated geometries like particulate bed but simply flat geometry. Figure 7 shows the visualized images of cupric ion behavior. In these images, the solid part appears to be black and the solution part is illustrated by gray color which becomes darker at the part with higher ion concentration. As seen in Fig. 7, the solution becomes denser in the vicinity of the upper reactive surface while it becomes more dilute in the vicinity of the lower surface. These concentration variations in the cell cause the gravitational instability near to the both surfaces and consequently finger-like convection is formed. Such convective flow was reported by de Bruyn (1995) in which the shadowgraphy is utilized to visualize the density variations of the ion.

\subsubsection{Shape effect of reactive interfaces on ion behavior}

We have examined ion behavior caused by gravitational instability accompanied with reaction at the complicated solid surfaces. Figure 8 shows the visualized images of cupric ion behavior at the initial stage of the deposition on three kinds of particulate bed (standard, comb-shaped and cove-shaped structures). In all cases, the finger-like downflow arises on the upper side (flat surface) and its behavior is similar to those shown in Fig. 7. It is found that the observed finger has almost the same size independently of the geometry of particulate bed and the size is comparable with the dominant wavelength $\lambda_{\max }=2 \pi / k_{\max }=1.694 \mathrm{~mm}$, which is calculated in the section 3.2.1. The dominant wavelength $\lambda_{\max }$ is shown in Fig. 8 and Fig. 9 by the length of a bar above the images.

On the other hand, the upward flow generated in the vicinity of the particulate bed indicates different behavior. Low-concentrated flow develops along the lower reacting surfaces as if it is immiscible with the surrounding denser fluid and it accumulates around the particle which is located at positionally higher point than 
the neighboring particles. As a result, streaky upward flow arises at the higher points of the particulate bed. The appearance of streaky upward flow implies that it is more stable for the fluid with low concentration to climb up the particles to the higher places than to form the finger-like flow at each location. In addition, it is suggested by comparison among the results for different geometries that the behavior of the upward flow greatly depends on the shape of the solid surfaces.

The upward flow interacts with the downward finger-like flow and finally the convection becomes steady state as shown in Fig. 9. It is found that the lengthscale of the convection in the cell is different depending on the solid surface geometry. For example, the convection lengthscale is almost the same as $\lambda_{\max }$ for the standard case while it is much greater for the cove-shaped case. These differences are attributed to the dependence of the streaky upward flow on the geometry of particulate bed. It is suggested that the ion transfer is not uniform and is dependent on the geometry of reactive solid-liquid interfaces. Such nonuniform ion transfer would be one factor causing the selective growth observed in the deposition experiments. However, the mechanism of the selective growth still needs to be discussed because the boundary flux at the interface is related to the electric potential as well as the ion concentration. It can be at least considered that the geometry of the interface has an influence on the mass transfer in overall system.

Figure 10 shows the average lengthscale of the downward, upward and steady convective flow for three kinds of surface structures. The results for downward flow quantitatively indicate that the lengthscale of the finger-like flow from flat surfaces is independent of the geometry of opposed particulate bed and it is similar to the predicted value $\lambda_{\max }$. It is also found from small error bars that the finger-like flow arises almost uniformly in space. On the other hand, the upward flow is extremely uneven and its lenghscale is sensitive to the interface geometry. The overall convection is formed under the influence of both upward and downward flow. Therefore, it is suggested that the resultant mass transfer is greatly influenced by the geometry of the reactive solid-liquid interface. 


\section{Conclusions}

Mass transfer in porous media with reactive solid-liquid interface is examined by electrochemical deposition experiment on particulate bed. The results showed that the overall ion transfer greatly depends on the structure of particulate bed, namely the surface geometry at the end. Moreover, the convective behavior of the cupric ion caused by the gravitational instability is visualized. It is found that the gravitational instability causes convection flow and it significantly varies the transport characteristics. The overall convection is formed under the influence of both upward and downward flow which are dependent on the interface geometry. It is suggested that the structure of the reactive solid-liquid interface greatly affects overall mass transfer.

This work was supported by Grant-in-Aid for JSPS Fellows 23·4066.

\section{References}

Békri S, Thovert JF, Adler PM (1995) Dissolution of porous media. Chem Eng Sci 50(17):2765-2791.

Borisova EA, Adler PM (2005) Deposition in porous media and clogging on the field scale. Phys Rev E 71:016311.

Colón CFJ, Oelkers EH, Schott J (2004) Experimental investigation of the effect of dissolution on sandstone permeability, porosity, and reactive surface area. Geochim Cosmochim Acta 68(4):805-817.

de Bruyn JR (1995) Fingering instability of gravity currents in thin-layer electrochemical deposition. Phys Rev Lett 74(24):4843-4846.

Ennis-King J, Paterson L (2007) Coupling of geochemical reactions and convective mixing in the long-term geological storage of carbon dioxide. Int J Greenh Gas Control 1:86-93.

Fernandez J, Kurowski P, Limat L, Petitjeans P (2001) Wavelength selection of fingering instability inside Hele-Shaw cells. Phys Fluids 13:3120-3125.

Harada S, Mitsui T, Sato K (2012) Particle-like and fluid-like settling of a stratified suspension. Eur Phys J E 35(1):1-6.

Hinch EJ, Bhatt BS (1990) Stability of an acid front moving through porous rock. J Fluid Mech 212:279-288.

Li X, Huang H, Meakin P (2008) Level set simulation of coupled advection-diffusion and pore structure evolution due to mineral precipitation in porous media. Water Resour Res 44:W12407. 
Newman J, Thomas-Alyea KE (2004) Electrochemical Systems 3rd ed. John Wiley \& Sons, New York.

Nicoli M, Castro M, Cuerno R (2008) Unified moving-boundary model with fluctuations for unstable diffusive growth. Phys Rev E 78:021601.

Riaz A, Hesse M, Tchelepi HA, Orr FM (2006) Onset of convection in a gravitationally unstable diffusive boundary layer in porous media. J Fluid Mech 548:87-111.

Rosso M, Chazalviel J-N, Fleury V, Chassaing E (1994) Experimental evidence for gravity induced motion in the vicinity of ramified electrodeposits. Electrochim Acta 39(4):507-515.

Sahimi M, Gavalas GR, Tsotsis TT (1990) Statistical and continuum models of fluid-solid reactions in porous media. Chem Eng Sci 45(6):1443-1502.

Sallès J, Thovert JF, Adler PM (1993) Deposition in porous media and clogging. Chem Eng Sci 48(16):2839-2858.

Schechter RS, Gidley JL (1969) The change in pore size distribution from surface reactions in porous media. AIChE J 15(3):339-350.

Völtz C (2003) Granular dynamics of density profiles in a suspension interface. Phys Rev E 68:021408. 


\section{Figure captions}

Fig. 1 Conceptual illustration of our system

Fig. 2 Experimental setup

Fig. 3 Absorption curve of cupric ion

Fig. 4 Change in surface shape of various particulate beds before and after deposition experiment (first and second row, respectively) and contourline of the surface height every 12 hours (third row)

Fig. 5 Ratio of liquid to solid parts at each height $h$ in the cell for (a) standard structure, (b) comb-shaped structure and (c) cove-shaped structure

Fig. 6 Relation between wave number and growth rate

Fig. 7 Convective behavior caused by gravitational instability

Fig. 8 Convective behavior of cupric ion at the initial stage; (I) $t=13 \mathrm{~s}$, (II) $t=16 \mathrm{~s}$, (III) $t=19 \mathrm{~s}$ and (IV) $t=22 \mathrm{~s}$

Fig. 9 Convective behavior of cupric ion at the steady state

Fig. 10 Convective lengthscales with various surface geometries 


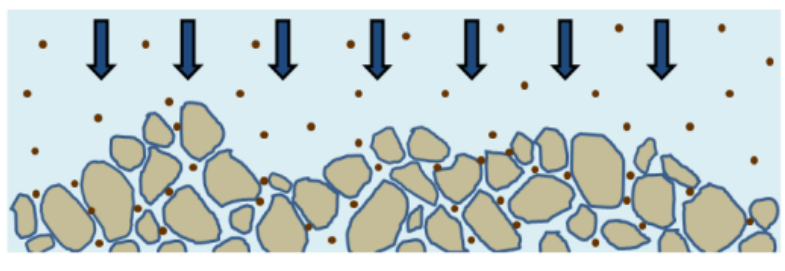

(a) initial state

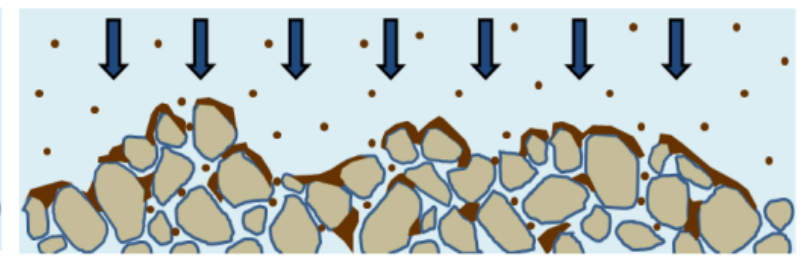

(b) after a passage of time

Fig. 1

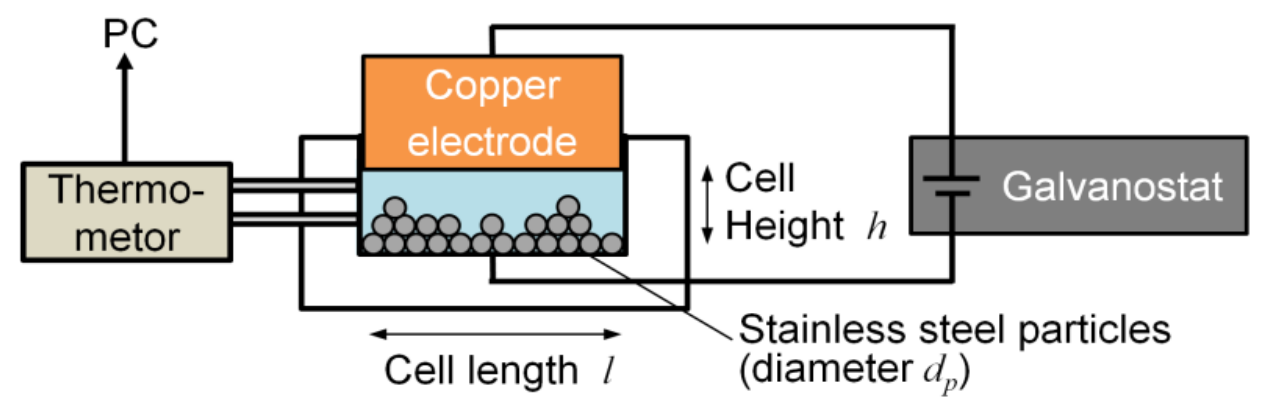

(a) electrodeposition cell

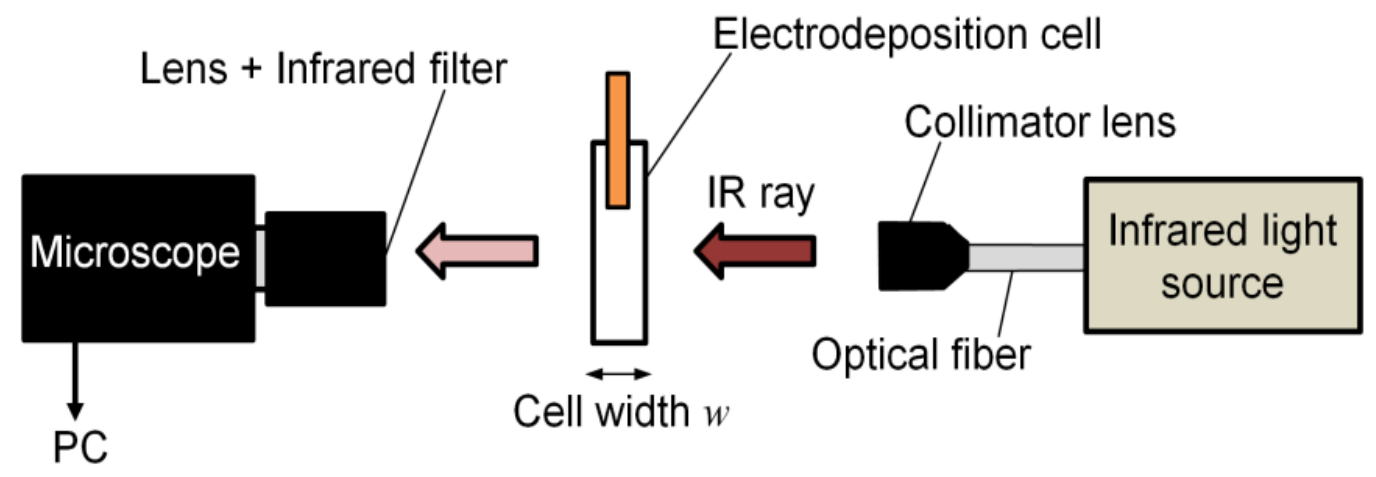

(b) apparatus for visualization

Fig. 2 


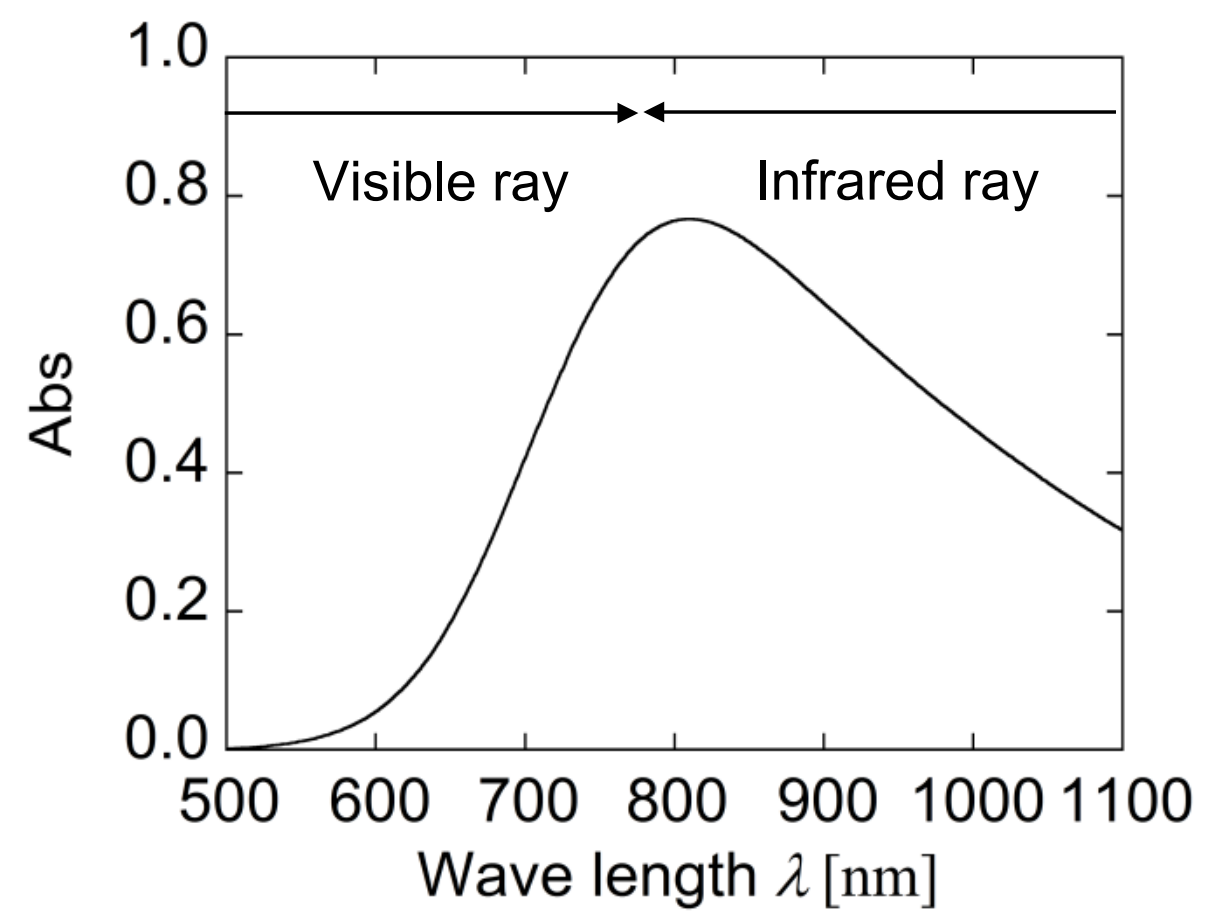

Fig. 3
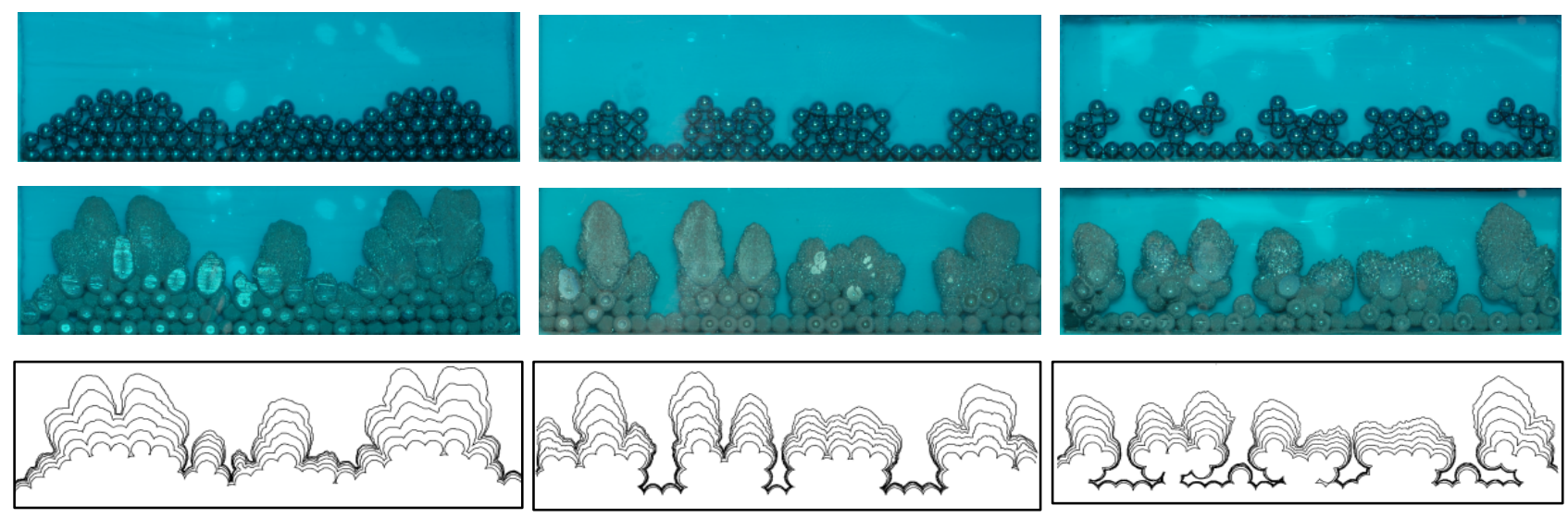

(a) standard structure

(b) comb-shaped structure

(c) cove-shaped structure

Fig. 4 


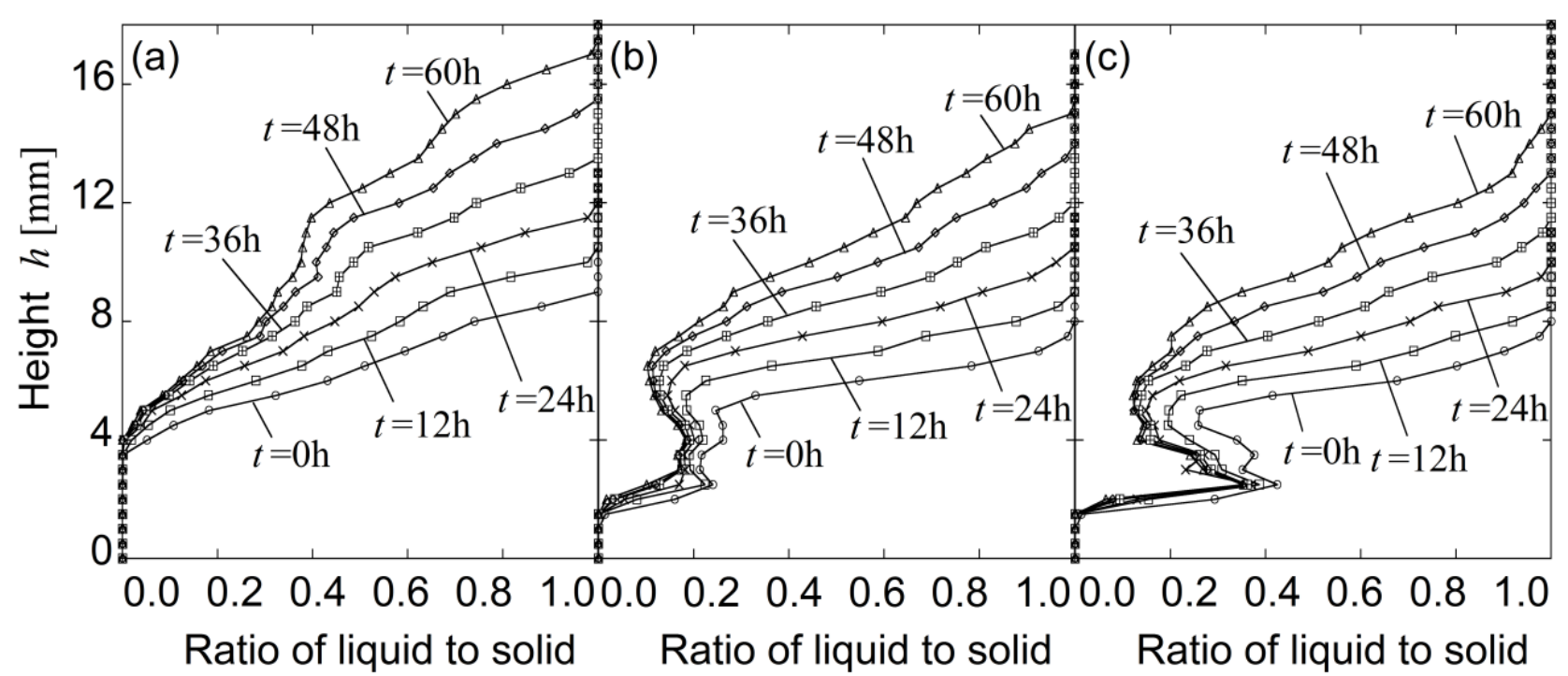

Fig. 5

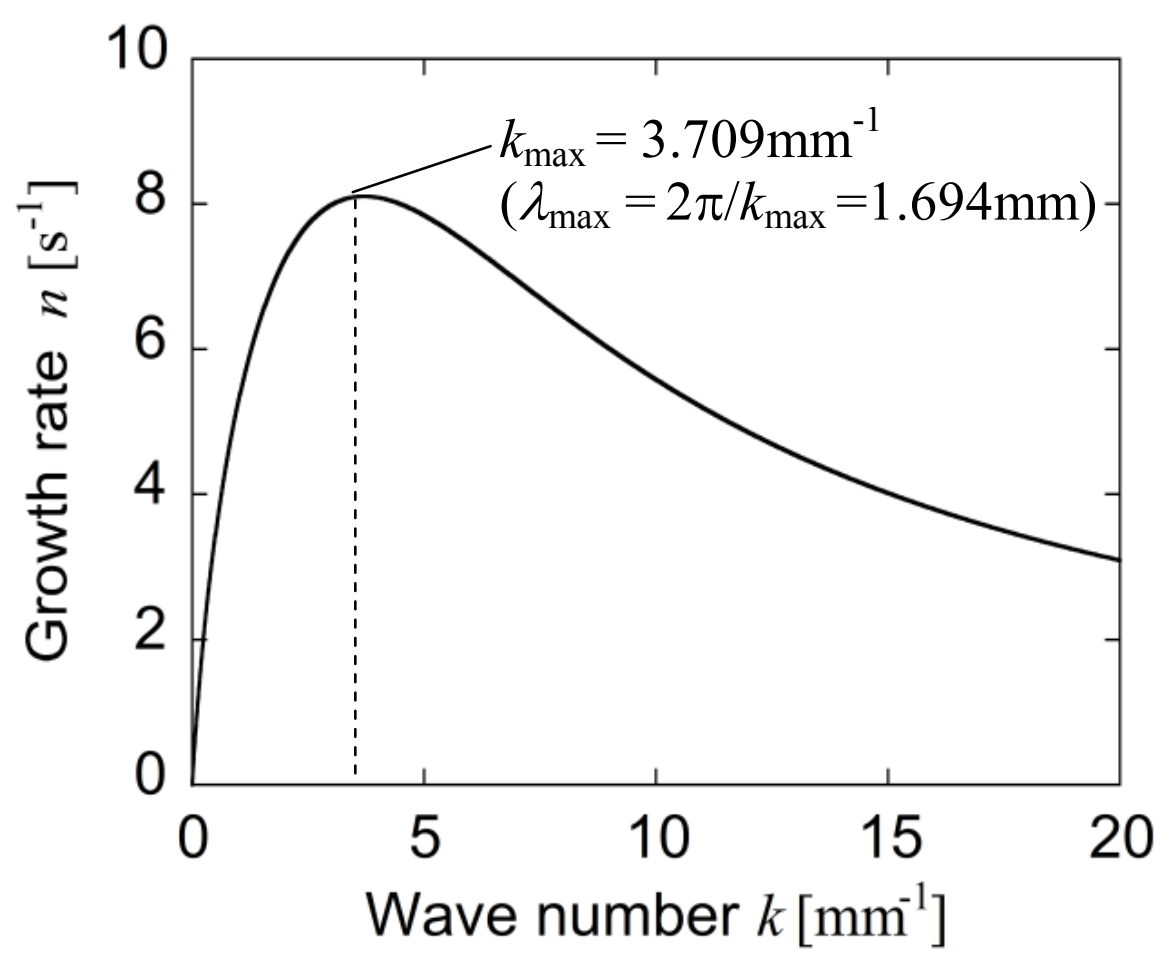

Fig. 6 


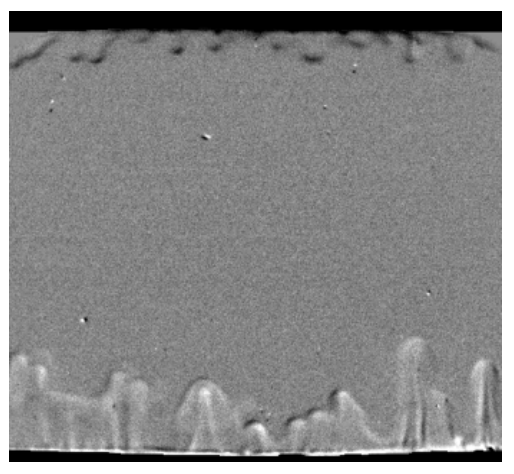

(I) $t=8 \mathrm{~s}$

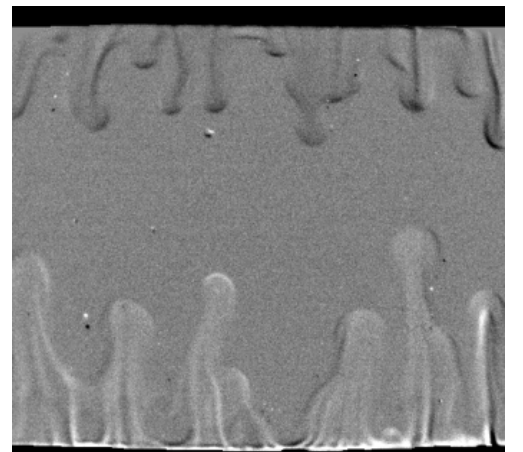

(II) $t=14 \mathrm{~s}$
Q113

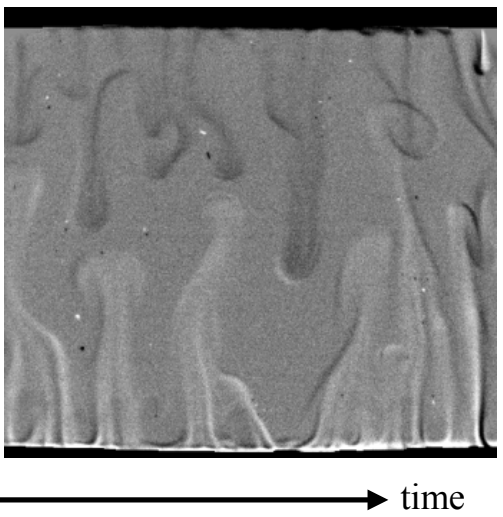

(III) $t=20 \mathrm{~s}$

Fig. 7 


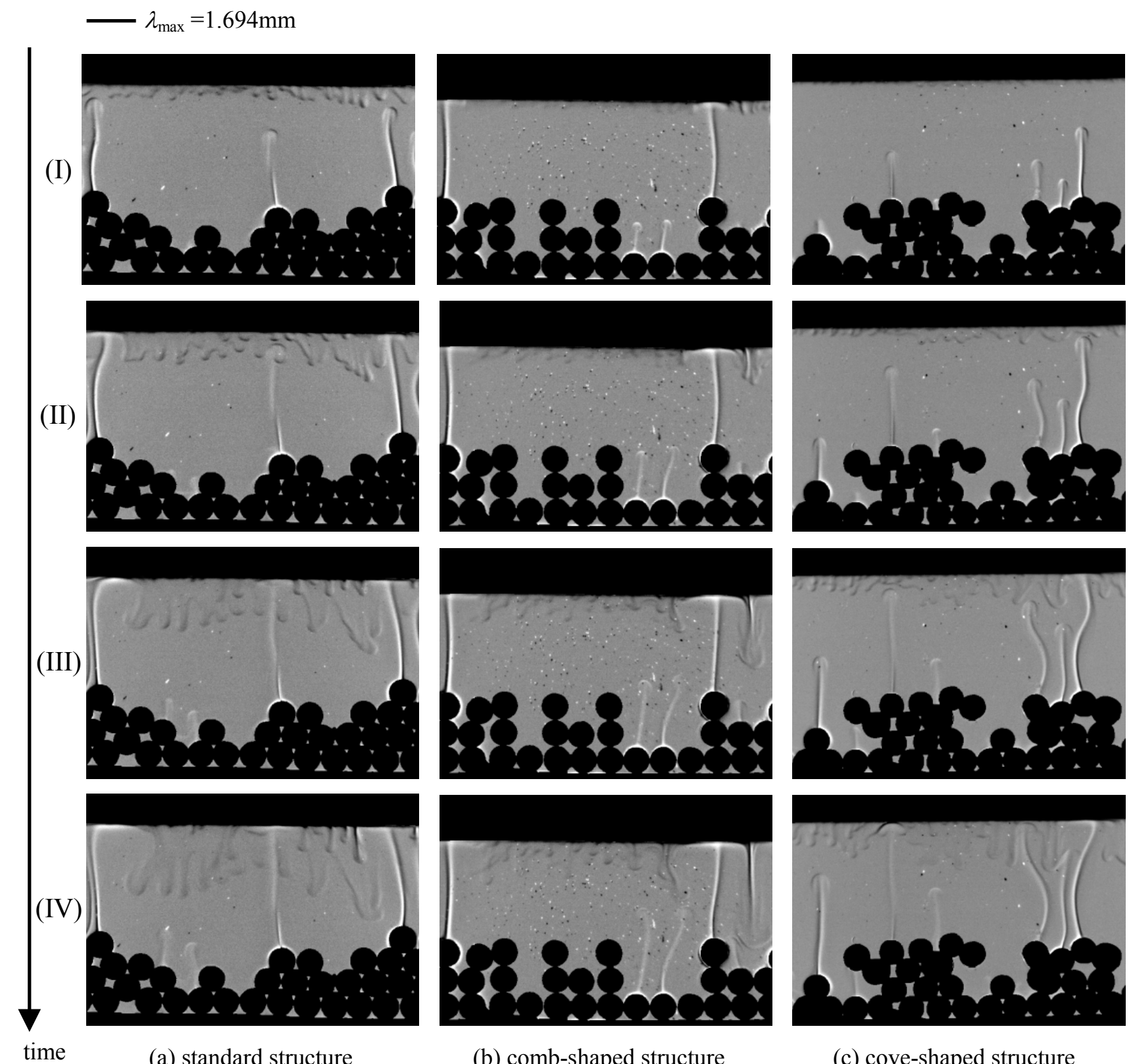

Fig. 8 


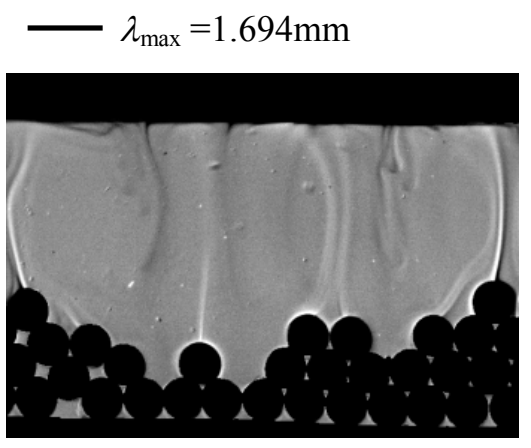

(a) standard structure

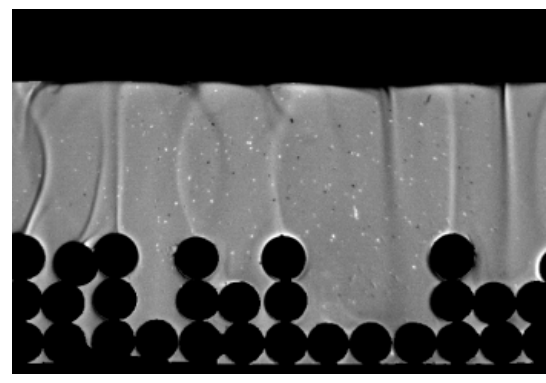

(b) comb-shaped sructure

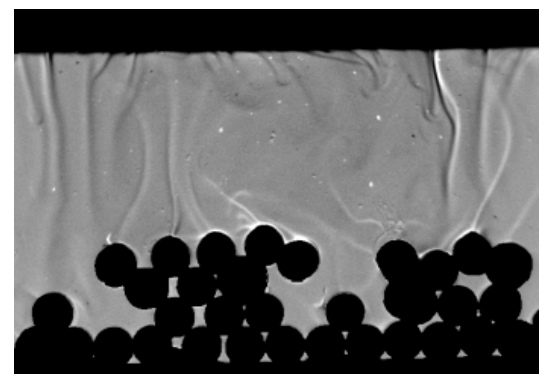

(c) cove-shaped structure

Fig. 9

Downward flow lengthscale

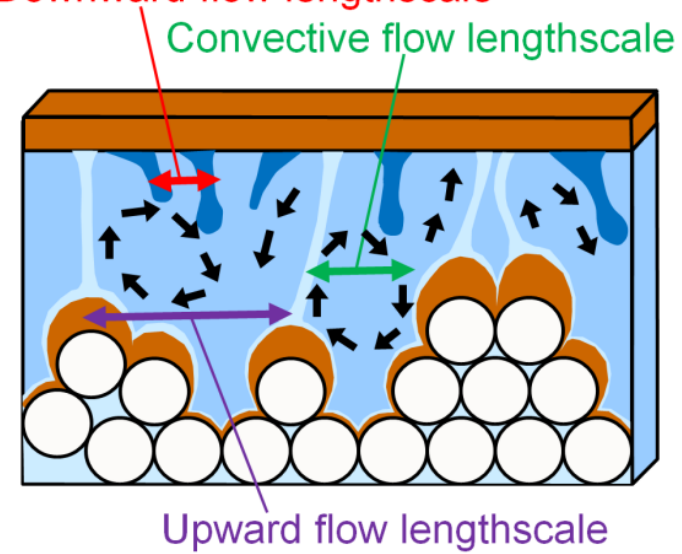

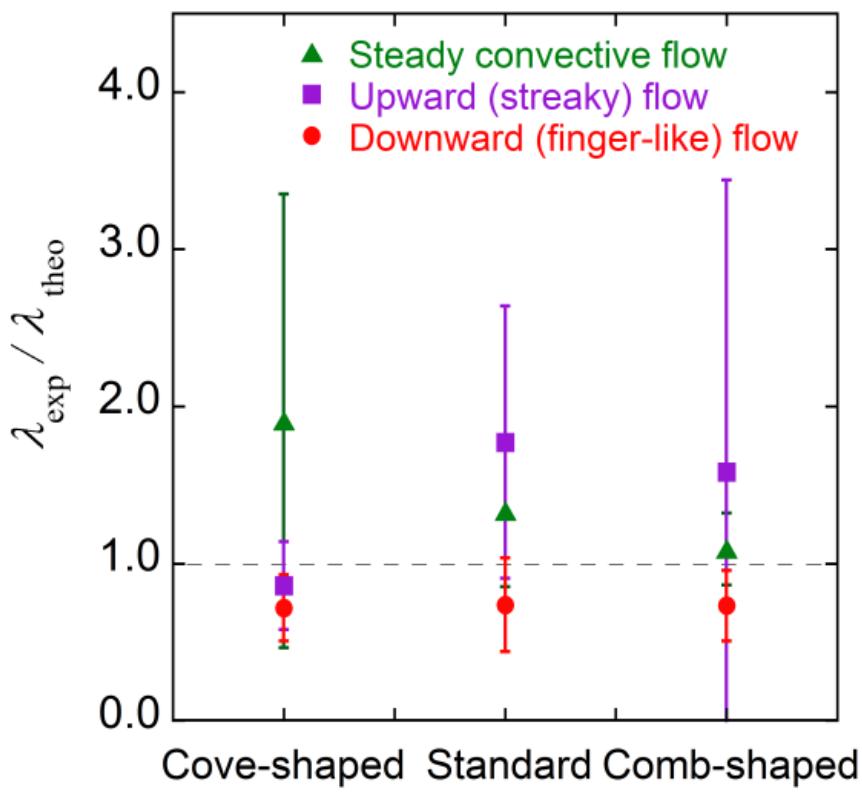

Fig. 10 